\title{
Correlating Atom Probe Tomography with Atomic-Resolved Scanning Transmission Electron Microscopy: Example of Segregation at Silicon Grain Boundaries
}

\author{
Andreas Stoffers, ${ }^{1,2, *}$ Juri Barthel, ${ }^{3,4}$ Christian H. Liebscher, ${ }^{2}$ Baptiste Gault, ${ }^{2}$ \\ Oana Cojocaru-Mirédin, ${ }^{1,2}$ Christina Scheu, ${ }^{2}$ and Dierk Raabe ${ }^{2}$ \\ ${ }^{1}$ Institute of Physics (IA), RWTH Aachen University, Otto-Blumenthal-Straße, 52074 Aachen, Germany \\ ${ }^{2}$ Max-Planck-Institut für Eisenforschung GmbH, Max-Planck-Straße 1, 40237 Düsseldorf, Germany \\ ${ }^{3}$ Central Facility for Electron Microscopy, RWTH Aachen University, Ahornstraße 55, 52074 Aachen, Germany \\ ${ }^{4}$ Ernst Ruska-Centre for Microscopy and Spectroscopy with Electrons, Forschungszentrum Jülich GmbH, 52425 Jülich, Germany
}

\begin{abstract}
In the course of a thorough investigation of the performance-structure-chemistry interdependency at silicon grain boundaries, we successfully developed a method to systematically correlate aberration-corrected scanning transmission electron microscopy and atom probe tomography. The correlative approach is conducted on individual APT and TEM specimens, with the option to perform both investigations on the same specimen in the future. In the present case of a $\Sigma 9$ grain boundary, joint mapping of the atomistic details of the grain boundary topology, in conjunction with chemical decoration, enables a deeper understanding of the segregation of impurities observed at such grain boundaries.
\end{abstract}

Key words: correlative microscopy, atom probe tomography, scanning transmission electron microscopy, grain boundary, silicon

\section{INTRODUCTION}

Macroscopic properties of materials depend on structure and chemical composition at the atomic scale. Thus, knowledge of the exact atomistic structure, and the corresponding compositional state of interfaces, is the ambitious goal of many researchers in materials science who seek deeper understanding of corresponding structurechemistry-performance interdependencies (Kuzmina et al., 2015). In the course of investigating these relations in multicrystalline silicon (mc-Si), we have developed a method to correlate high-resolution scanning transmission electron microscopy (HRSTEM) with atom probe tomography (APT). Both techniques have already been used individually to analyze the atomic structure of Si grain boundaries (GBs) (Couillard et al., 2013) and the distribution of impurities and dopants in Si (Thompson et al., 2005, 2007a; CojocaruMirédin et al., 2009; Philippe et al., 2009; Blavette et al., 2014). Several early and also advanced approaches already exist to correlate results from APT and transmission electron microscopic (TEM) measurements (Thuvander et al., 1996; Herbig et al., 2014; Rigutti et al., 2014; Kuzmina et al., 2015; Lefebvre et al., 2015; Weber et al., 2016), however, the direct and joint mapping of atomically resolved STEM information and APT data of an interface has not been yet demonstrated.

Mc-Si is still the dominant absorber material used for solar cell fabrication. Compared with monocrystalline Si, it

Received June 29, 2016; accepted December 26, 2016

*Corresponding author. stoffers@mpie.de contains a high amount of impurities and defects, such as dislocations and GBs, increasing the recombination activity and thus reducing cell efficiency (Kveder et al., 2001; Di Sabatino \& Stokkan, 2013). We recently published chemical details of different recombination active GBs using joint electron beam induced current (EBIC) and electron backscatter diffraction (EBSD) experiments and APT (Stoffers et al., 2015a). In that case, we were only able to estimate the details of the GB structure, such as coherency at the micron scale, based on the EBSD data alone, neglecting details at the nano- or atomic scale. However, what the atomic arrangements actually look like for the different GB types and how these structures influence the impurity segregation are still unanswered questions. In a subsequent study, we have already resolved the high complexity of the atomic structure of one of the currently studied GBs by HRSTEM (Stoffers et al., 2015b). We will discuss in this manuscript the combination of APT and HRSTEM and how this approach promotes the interpretation of the APT data.

\section{Materials}

$\mathrm{Mc}-\mathrm{Si}$ ingots were grown by directional solidification in a $200 \mathrm{~mm}$ crucible. In order to study the influence of the most detrimental transition metals, the ingots were intentionally contaminated with $20 \mathrm{ppm} \mathrm{Fe}$ and $20 \mathrm{ppm} \mathrm{Cu}$ during the solidification process to increase the driving force for GB segregation (Riepe et al., 2011; Stoffers et al., 2015a). Samples analyzed in the present study were taken from a thin 
wafer extracted from the capping area of the ingot at $180 \mathrm{~mm}$ height, where the concentration of impurities is known to be the highest. The samples were precharacterized by EBIC and EBSD to map the electrical activity of the present GBs together with the orientation relationships of the corresponding grains. In some cases, performing a detailed trace analysis based on the EBSD data can allow a global GB plane to be extracted (Stoffers, et al., 2015a). By the term "global GB plane" we refer to the fact that the actual interface planes of GBs can, in certain cases, decompose further into nanoscale facets, which cannot be detected by EBSD maps and so their exact topological analysis requires the use of HRSTEM.

\section{Methods}

Aberration-corrected STEM allows the visualization of the local arrangement of single atomic columns, with the column intensities depending on the projected mean atomic number, which is an intuitive way to study the atomistic structure of small features and interfaces in two dimensions (2D). The optimum tool for a space-resolved analysis of the chemical composition in $3 \mathrm{D}$ at the nanoscale is APT. For a correlative approach, it is of highest interest, to analyze the structure and the chemistry at the same location or at least two locations that are very close together. This can be realized by two different approaches: (1) APT and HRSTEM are performed on the same specimen. As APT requires a tip-shaped specimen, this can only be implemented by conducting the HRSTEM directly on the APT tip. (2) APT and HRSTEM are performed on individual samples stemming from the same interface with a minimum lateral separation. The first approach has the advantage of enabling a true 1:1 correlation. However, the specimen has a tip shape with increasing thickness along the tip axis, quickly resulting in non-ideal conditions for HRSTEM. Furthermore, the examinable volume for structural analysis is drastically reduced compared with a conventional TEM lamella. Therefore, we mainly focus on the second approach here, but we also briefly report one example where the first approach has been applied toward the end of this manuscript.

In order to extract samples for APT and TEM analysis from closely spaced positions, we established a combination of the site-specific lift-out method for preparing APT specimens (Felfer et al., 2012) with the plan-view lift-out method for TEM lamellae (Langford et al., 2001), using a focused ion beam (FIB). A schematic view of the sample volume lifted out is sketched in Figure 1. In this example, the samples are prepared from the area around a triple junction (TJ), obtaining a TEM foil containing the TJ along with two series of three APT specimens containing the two of the GBs on the right and left side of the lamella. Both the TEM sample and the APT specimens, are prepared parallel to the sample surface, enabling a direct correlation with the EBIC and EBSD information generated from the sample surface. Furthermore, the EBSD data were used to select appropriate regions, where all grains have a common zone axis direction close to the normal of the sample surface $\left(<15^{\circ}\right)$ and where the GBs can be viewed "edge-on". This is essential for reaching atomic resolution across the GBs in HRSTEM. The size of the milled trench determines the maximum dimensions of the TEM lamella and in this study we milled trenches around a $30 \times 3 \mu \mathrm{m}^{2}$ large area, resulting in a $3 \mu \mathrm{m}$ wide TEM lamella. According to the adopted lift-out methods (Langford et al., 2001; Felfer et al., 2012), the volume is then attached to a micromanipulator by means of platinum deposition inside the FIB. Three small wedgeshaped portions of the right-hand GB are subsequently extracted and attached to the horizontally aligned supporting tips of a TEM half grid. Subsequently, the bigger portion for TEM lamella is glued to a conventional OmniProbe grid in the same way, followed by extraction of three wedges from the GB on the left-hand side. After mounting all these wedges, both grids are flipped by $90^{\circ}$ into a vertical orientation for the milling process. A custom-designed sample retainer was used (Herbig et al., 2015), which enables mounting and flipping of the grids in the different microscopes and thus minimizing the risk of damaging the samples during the preparation process. The APT specimens are then prepared in the conventional way by annular milling (Thompson et al., 2007b), with additional imaging of the GB position by transmission Kikuchi diffraction (TKD) in between the milling steps (Babinsky et al., 2014). Likewise, the TEM foil is prepared by FIB milling in the conventional way (Langford et al., 2001). Here, the milling is mainly performed on the thinner side of the wedge-shaped volume, to retain a large lamella surface and to speed up the milling process. In both cases, the energy of the ion beam is stepwise reduced during the milling process. Trenches are milled at $30 \mathrm{kV}$, and most of the milling is performed at $16 \mathrm{kV}$. Gentle milling at the later stages is performed at $8 \mathrm{kV}$, and final cleaning is performed at $2 \mathrm{kV}$ to achieve a high surface quality (Schaffer et al., 2012). The success rate of the FIB-based sample preparation strongly depends on the user's experience and was in the range of $75 \%$ in our case.

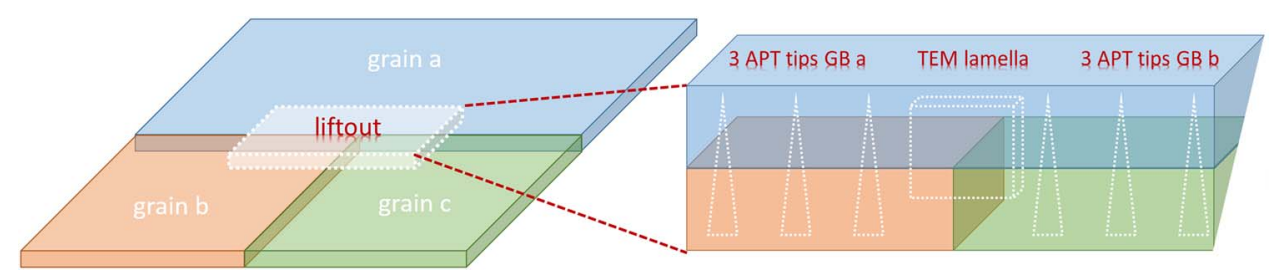

Figure 1. Schematic view of lift-out idea. 
A great improvement of the success rate was achieved by additional TKD measurements in between the final milling steps. This enables the monitoring of the GB position with an adequate precision $(\sim 10 \mathrm{~nm})$ and thus avoids blind milling (GBs are not visible in the SEM).

FIB milling and EBSD were performed on a FEI (Hillsboro, OR, USA) Helios Nanolab 600 dual beam FIB. The acceleration voltage of the electron beam was set to $10 \mathrm{kV}$ for EBSD and $15 \mathrm{kV}$ for TKD, and an electron probe current of $1.4 \mathrm{nA}$ was used in both cases. The HRSTEM high-angle annular dark field (HAADF) images were recorded with a FEI Titan 80-200 electron microscope operated at $200 \mathrm{kV}$ and a probe-corrected FEI Titan Themis 60-300 electron microscope operated at $200 \mathrm{kV}$. APT measurements were performed on a LEAP3000 X HR and a LEAP5000 XS (CAMECA Instruments, Madison, WI, USA), enabling highest ion detection efficiency ( 80\%). Accordingly, the laser wavelengths used were 532 and $355 \mathrm{~nm}$, and pulse energies were set to 300 and $30 \mathrm{pJ}$, respectively.

\section{Results}

A first example is presented in Figure 2. The recombination activity of the analyzed sample area (Fig. 2a) is compared with the GB coincident site lattice (CSL) character extracted from the EBSD measurements in Figure 2b. A TEM lamella of the highlighted TJ was prepared using the described method. An HAADF STEM image is presented in Figure 2c resolving the atomic structure in the direct vicinity of the TJ. All three grains are viewed along $<110\rangle$ zone axis, making the typical Si dumbbells observable in all grains at the same time (Dabrowski \& Müssig, 2000; Batson et al., 2002). This allows us to assign each dumbbell to one of the three grains based on its orientation. In addition to the orientation of the three present grains indexed in RGB in Figure 2b, two more dumbbell orientations were observed at the two non- $\Sigma 3$ interfaces (orange ellipses), together with single atomic columns (yellow circles).
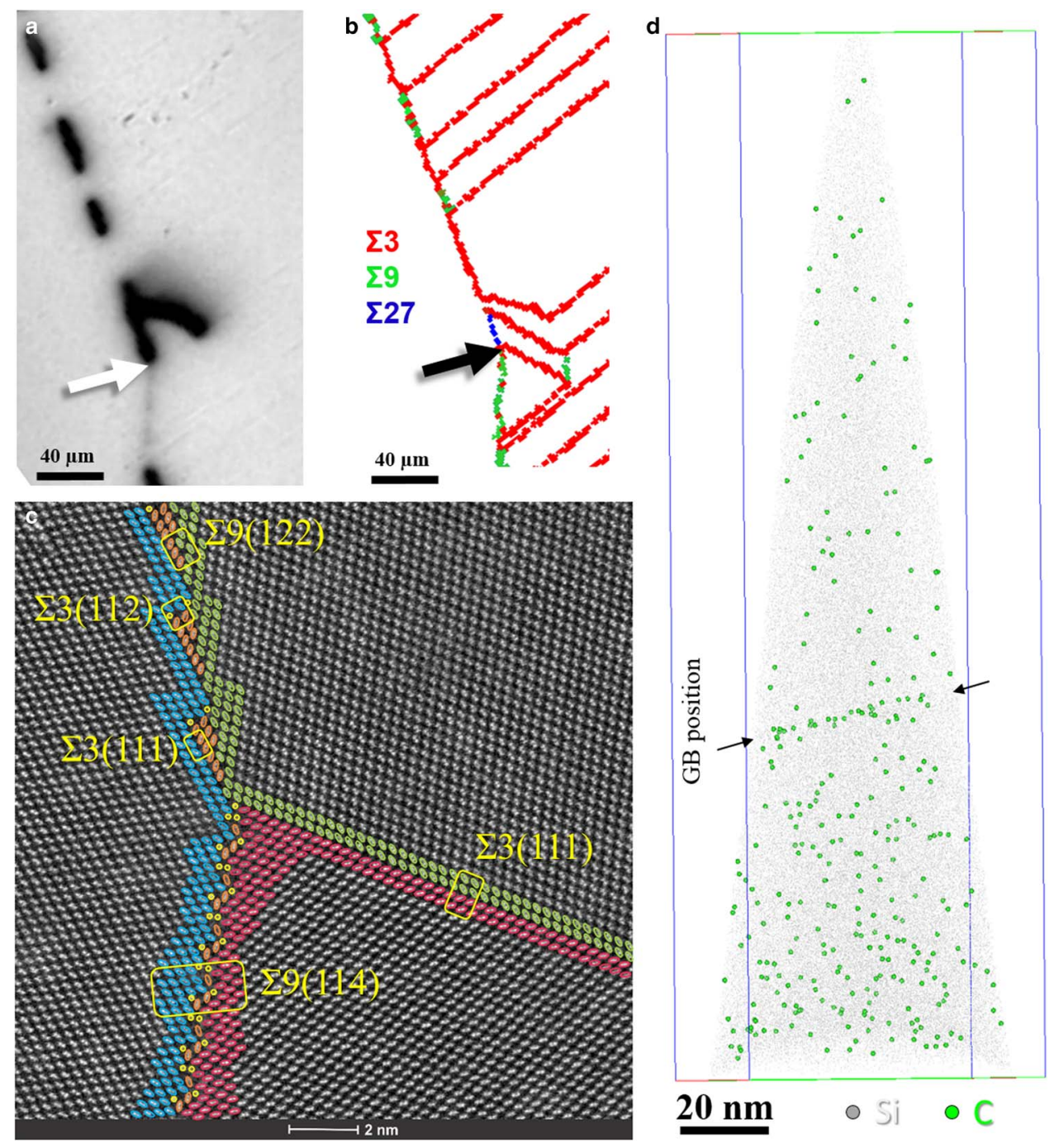

Figure 2. EBIC map (a) and corresponding CSL character of present GBs (b) extracted from EBSD scan with position marked for further analysis (white arrow in a, black arrow in b). Corresponding triple junction in HAADF STEM resolving the atomic arrangement of the different GBs (c). Corresponding APT measurement (LEAP 3000 HR) of $\Sigma 27$ GB segment showing little C segregation (d). 
The HAADF STEM image can already provide highly interesting structural details without further extensive simulations. The present $\Sigma 3$ tilt GB shows nearly perfect structure of a coherent and symmetric twin boundary. The term "coherent" in this context refers to the fact that one atomic layer belongs consistently to both adjacent grains. The Si dumbbells are rotated by $70^{\circ}$ and the GB plane is (111) with respect to both crystals. Following the trace of the GB, no change in this nearly perfect symmetry was observed.

The atomic structure observed along the $\Sigma 9$ GB is much more complex. Its structural motifs are repeated periodically, with a length of its underlying structural units of $1.5 \mathrm{~nm}$, over a distance of $>20 \mathrm{~nm}$. A single structural unit is highlighted by the yellow frame. The complex atomic structure makes it difficult to assign a distinct GB plane direction, but in agreement with the expectations from trace analysis performed on the EBSD measurements (not shown here), the GB plane is close to (114). The misorientation of both crystals measured in the HAADF micrograph is $36 \pm 1^{\circ}$, whereas a misorientation angle of $39.0 \pm 0.5^{\circ}$ was determined by means of EBSD. The small deviation from the ideal $\Sigma 9$ misorientation $\left(38.94^{\circ}\right)$ in the HAADF micrograph falls well within the Brandon criterion (Brandon, 1966), and may in addition be altered by the presence of scan distortions and sample drift.

The atomic structure of the present $\Sigma 27$ GB reveals a feature to compensate for non-ideal GB properties. Here, the misorientation of the two crystals is $33 \pm 1^{\circ}$ in the HAADF micrograph and $31.7 \pm 0.5^{\circ}$ in the EBSD measurement, which is close to the ideal misorientation of $31.58^{\circ}$. The global GB plane could not be identified by the trace analysis in this case, indicating that the global GB plane is asymmetric. The HAADF image in Figure $2 c$ confirms the asymmetry and reveals the faceted atomic structure of this interface. A set of nanograins with a diameter of $3 \mathrm{~nm}$ and a third orientation is introduced (orange ellipses). This results in dissociation of the $\Sigma 27$ interface into numerous triangular shaped grains bordered by symmetrical $\Sigma 3, \Sigma 3$, and $\Sigma 9$ interfaces: $\Sigma 27 \rightarrow \Sigma 3(111)+\Sigma 3(112)+\Sigma 9(122)$.

The corresponding EBIC map in Figure 2a shows a strong recombination activity at the $\Sigma 27$ interface, whereas the recombination activity at the $\Sigma 3$ and $\Sigma 9$ interfaces are negligible. This difference may be caused by the observed GB structure of the $\Sigma 27$ interface or by additional impurity segregation. APT analysis of that interface is shown in Figure $2 \mathrm{~d}$ and reveals a slight $\mathrm{C}$ segregation at the position of the GB in the 3D ion map. The presented APT volume was reconstructed on the basis of the tip profile observed in SEM, using the corresponding reconstruction routine in the IVAS $^{\varpi}$ software package. APT analysis of the $\Sigma 9$ interface was not successful, but previous work on recombination inactive interfaces revealed no impurity segregation (Ohno et al., 2013; Stoffers et al., 2015a). The GB dissociation at the $\Sigma 27$ interface most probably promotes the observed segregation of $\mathrm{C}$ and thus increases the recombination activity. This implies a significant influence of $\mathrm{C}$ segregation on the GB recombination activity, in agreement with our own work (Stoffers et al., 2015a) and other researchers (Pizzini et al., 2005).

A second example of a GB analyzed by the correlative approach is shown in Figures 3-6. In this case the correlative approach was used to study a single $\Sigma 9$ interface with a higher recombination activity than the $\Sigma 27$ interface discussed above. Figure 3 shows the EBIC map (a), GB CSL character extracted from the EBSD measurements (b) together with an SEM/TKD overlay image of the corresponding TEM lamella (c), and APT specimen (d). The grains appear nearly in the same color in the TKD map, as both grains are oriented close to the $\langle 110\rangle$ zone axis. The GB position (white line) is highlighted by the white arrows. The misorientation of both crystals measured in the HAADF micrograph in Figure 4 is $38 \pm 1^{\circ}$, which is very close to the ideal misorientation of $38.94^{\circ}$. The observed atomic structure in this case is again very complex and shows a strong faceting of numerous $\Sigma 9$ segments with different GB habit planes. Several straight (114) and (122) facets of varying lengths in the range of $10 \mathrm{~nm}$ were observed. The interface structure observable in Figure 4 contains a straight (122) segment and a slightly bent segment close to (114), which consists of the same structural atomic motifs as observed in the first example in Figure 2c. Due to the strong curvature of the GB plane, the atomic structure is heavily distorted and no periodicity similar to the previous example (Fig. 2) was observed, although the same structural units can be found along the whole GB. The corresponding APT measurement is presented in Figures 5 and 6. The observed impurity decoration follows the GB interface, which consists of two segments within this APT specimen. Figure 5 a represents the complete APT measurement viewed along the common GB plane direction, where only $\mathrm{C}$ and $\mathrm{Si}$ were plotted. The wide field of view and high detection efficiency of the LEAP5000 XS system enables the clear observation of multiple crystallographic poles on the detector, as can be seen on the detector density maps in Figures $5 b$ and $5 c$ for the blue and red plane shown in (a). The (113) pole in the upper grain and the (111) pole in the lower grain were selected for optimization of the reconstruction parameters. Small volumes corresponding to the dashed circles were reconstructed to confirm correct lattice plane distances, as can be seen in Figures $5 \mathrm{~d}$ and $5 \mathrm{e}$.

In Figure 6, the 3D ion maps of the volume around the GB are plotted for the detected impurity species. These 3D maps nicely demonstrate the preferential segregation of different impurity species at the facets: $\mathrm{Fe}$ and $\mathrm{N}$ are detected in form of the complex $\mathrm{FeN}^{2+}$ at $m / z=35 \mathrm{Da}$ and decorate both interface segments. The detection as a complex ion does not necessarily imply that these species are segregating in a molecular form. Rather, it is likely that they are segregating individually. However, the atomic species Fe and $\mathrm{N}$ were not observed in the mass spectra, most probably due to peak overlap of $\mathrm{Fe}^{2+}$ with $\mathrm{Si}^{+}(28 \mathrm{Da})$ and $\mathrm{N}^{+}$with $\mathrm{Si}^{2+}(14 \mathrm{Da})$. Therefore, the given $(\mathrm{Fe}+\mathrm{N})$ concentration should be taken as a lower limit of the effectively segregated amount. 


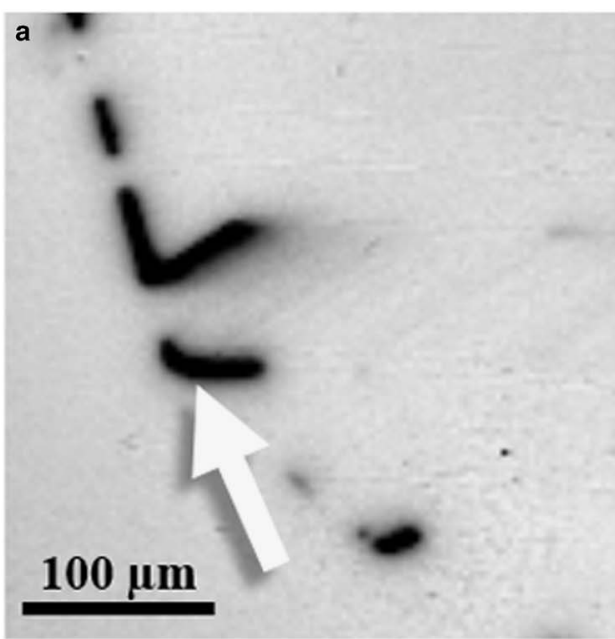

b
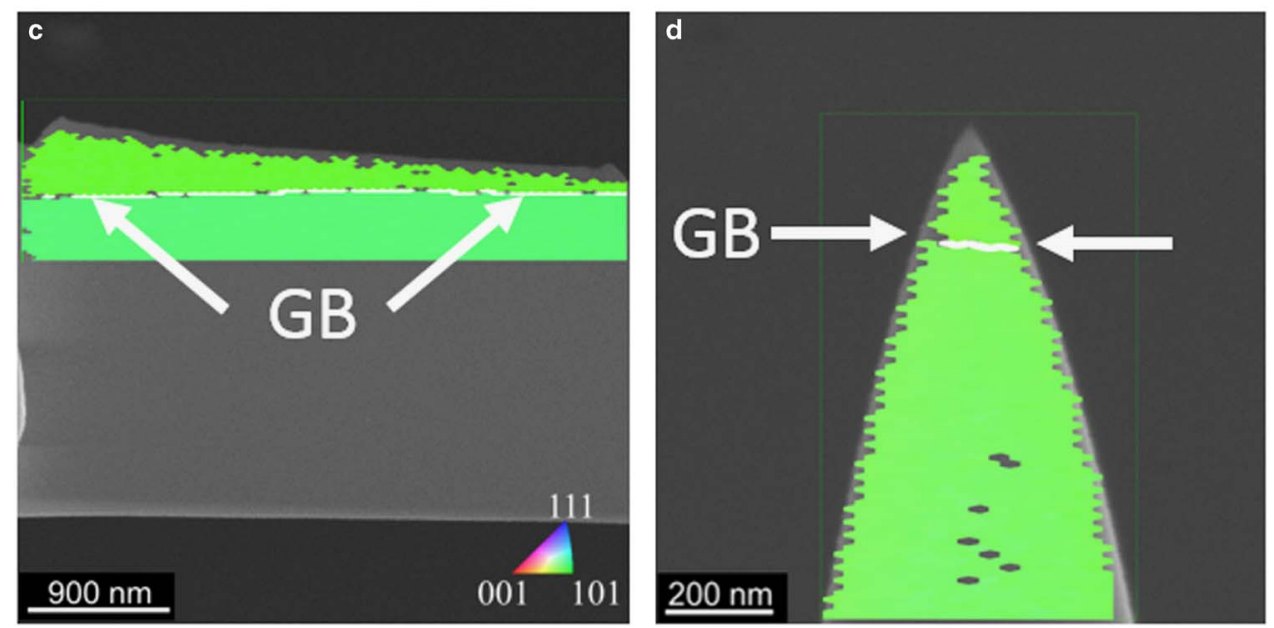

Figure 3. EBIC map (a) and corresponding CSL character of present GBs (b) extracted from EBSD scan with position marked for further analysis (white arrow in a, black arrow in b). SEM image superimposed with grain orientations from Transmission Kikuchi Diffraction of the corresponding TEM lamella (c) and an APT specimen (d) prepared using the described method. The GB (white line) is indicated by the white arrows.

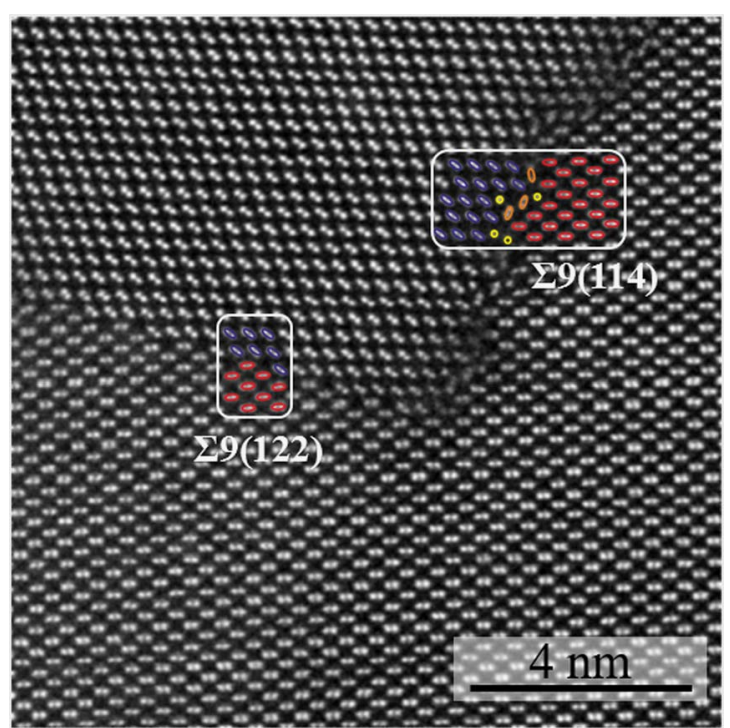

Figure 4. Observed atomic structure in the TEM lamella corresponding to the APT data presented in Fig. 5 and 6. The colored facet motifs are the same as observed in Fig. 2(c).
$\mathrm{SiO}^{+}$(44 Da) was only observed on the left segment (facet A) and $\mathrm{Cu}^{+}$can only be found along the right segment (facet $\mathrm{B}$ ). $\mathrm{O}^{+}$is detected only within a very small volume at the junction of both facets. To quantify the amount of segregated impurities, the Gibbsian interfacial excess for the observed impurity species was calculated (Krakauer \& Seidman, 1993) within three small sub-volumes representing facet A, facet B, and the junction of the two (kink). As can be seen in the summarized results in Table 1, the highest impurity segregation is found at the junction of the two facets. $(\mathrm{Fe}+\mathrm{N})$ is present at both facets in a similar amount, whereas $\mathrm{Cu}$ is only present in facet $\mathrm{B}$, and $\mathrm{O}$ is present in facet $\mathrm{A}$ with a higher Gibbs excess. The calculated excess values are comparable to the recently published results (Stoffers et al., 2015a). Transition metals, in particular, are known to drastically increase the recombination activity in $\mathrm{mc}-\mathrm{Si}$ (Istratov et al., 2003), suggesting that the detected $\mathrm{Cu}$ and Fe impurities are responsible for the strong EBIC signal in this case. Remarkable at this point, is the individual impurity uptake of different $\mathrm{GB}$ facets depending on their GB plane, as demonstrated here in a single measurement. 

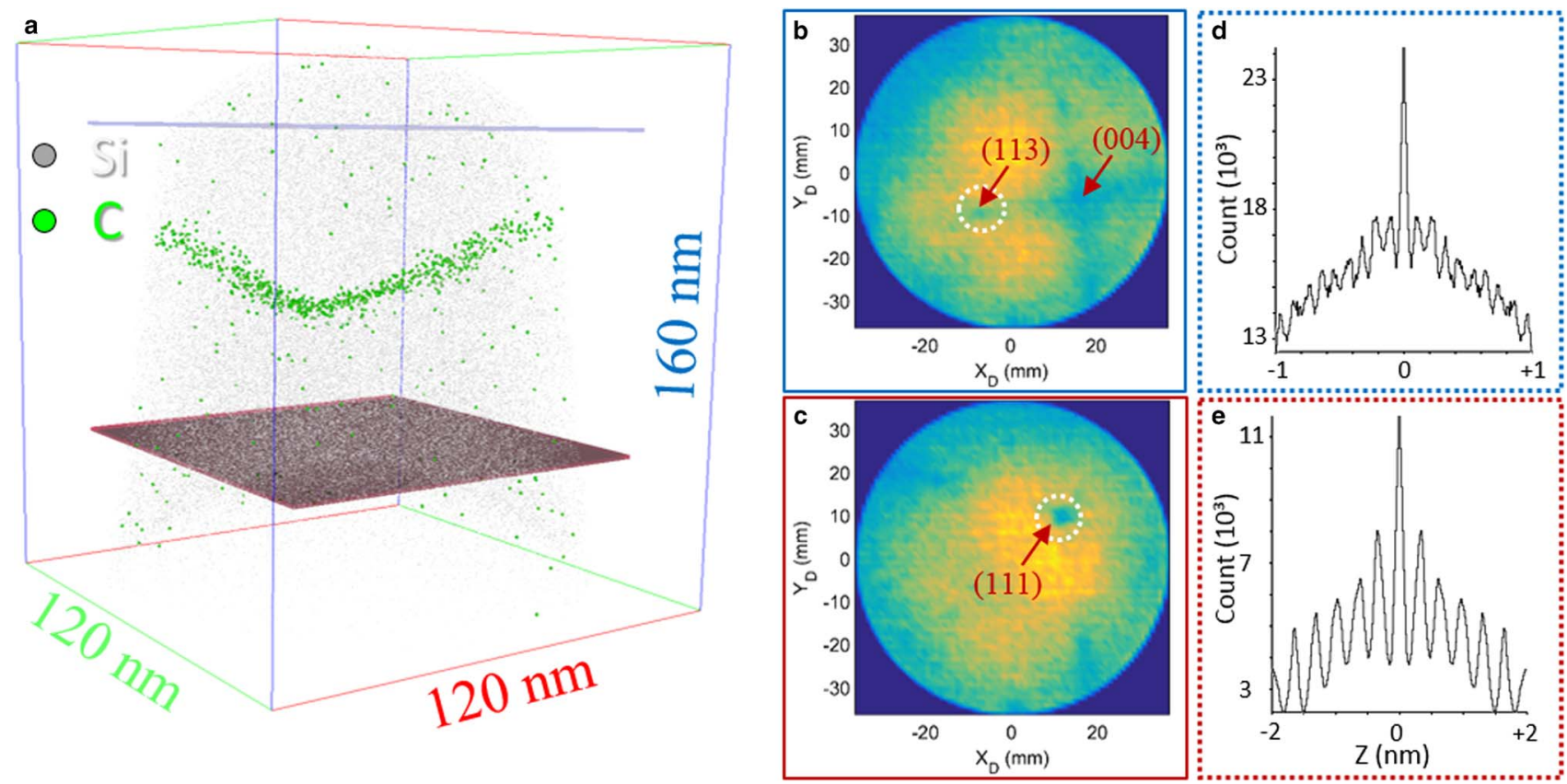

Figure 5. APT reconstruction (LEAP $5000 \mathrm{XS}$ ) showing C segregation along two different GB facets (a). Detector density maps were plotted at the position of blue plane in the upper grain $(\mathbf{b})$ and at the position of the red plane in the lower grain (c) enabling the identification of several pole directions. The APT reconstruction was optimized for the volume corresponding to the dashed circles until the lattice distances fitted the expected values (d, e).
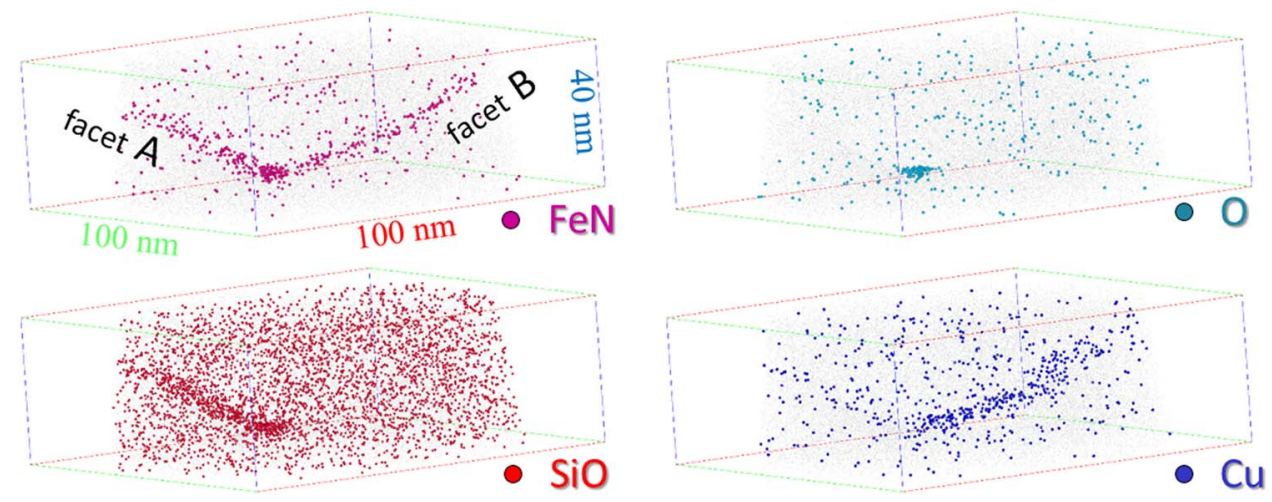

Figure 6. APT reconstruction (LEAP $5000 \mathrm{XS}$ ) showing strong differences in the segregation behavior of the observed impurity species at the two facets.

This phenomenon can now be interpreted in correlation to the HRSTEM data. The GB consists mainly of the two facets shown in Figure 4, which profoundly differ in atomic arrangement. Most likely, these differences in atomic structure influence the impurity segregation. The known directions of the $\langle 110\rangle$ zone axis in the APT specimen and the reconstructed GB planes, allow us to determine that facet $\mathrm{B}$

Table 1. Calculated Gibbs' interfacial excess values.

\begin{tabular}{|c|c|c|c|c|c|}
\hline at. $/ \mathrm{nm}^{2}$ & $\Gamma_{\mathrm{SiO}}$ & $\Gamma_{\mathrm{C}}$ & $\Gamma_{\mathrm{FeN}}$ & $\Gamma_{\mathrm{O}}$ & $\Gamma_{\mathrm{Cu}}$ \\
\hline Facet A & 0.17 & 0.08 & 0.04 & - & - \\
\hline Facet B & - & 0.09 & 0.02 & - & 0.03 \\
\hline Kink & 0.30 & 0.09 & 0.15 & 0.23 & 0.06 \\
\hline
\end{tabular}

most likely has a (122) habit plane and thus consists of the structure observed in Figure 4. Our observation in the present study suggests that segregation is sensitive to the local GB facet and thus to the local atomic arrangements. Preferential segregation might be caused by the fact that the (122) structure of facet B offers not much space for interstitial impurities. Hence, only substitutional $\mathrm{C}$ atoms and small amounts of interstitial $\mathrm{Cu}$ and $(\mathrm{Fe}+\mathrm{N})$ are segregating. In contrast, a relatively high amount of (interstitial) $\mathrm{O}$ impurities can be observed at facet $\mathrm{A}$, which seems to have much more complex atomic structure with a higher probability to offer open sites for impurity segregation. Furthermore, the different impurity species might mutually influence their segregation behavior. Recent computational work on GB segregation revealed complicated 


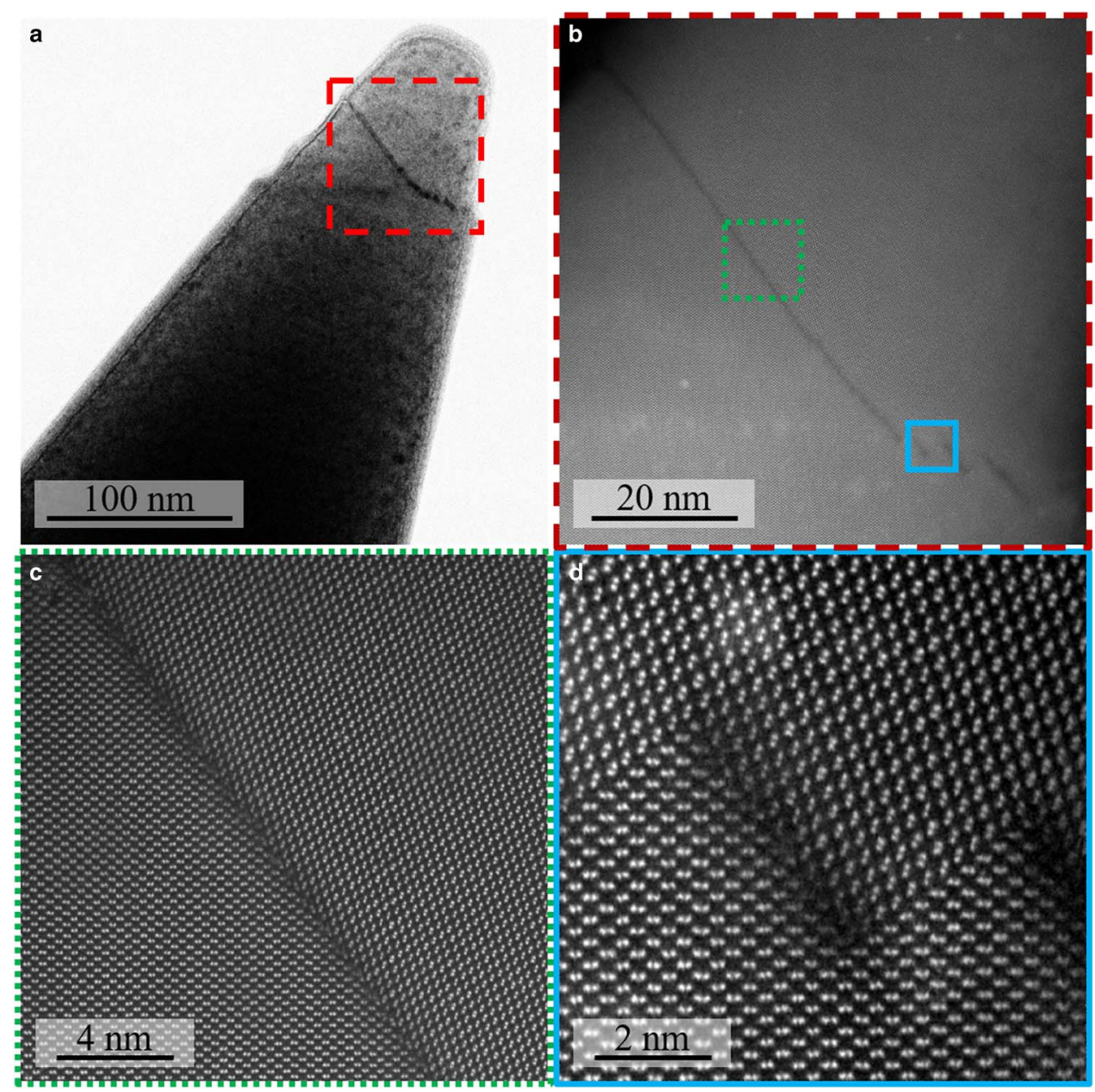

Figure 7. HAADF STEM images of a GB inside an APT specimen at different magnifications.

interdependencies between local strain, atomic coordination, and the atomic impurity segregation at the different GB types (Käshammer \& Sinno, 2015; Ziebarth et al., 2015). Accordingly, a more detailed understanding of the mechanisms leading to the observed preferential segregation would require extensive computational efforts, taking into account different local atomic structures and co-segregation effects of interstitial and substitutional impurities.

In order to achieve a true 1:1 correlation of atomicresolved STEM and APT it is necessary to perform both experiments exactly on the same specimen. In principle, this kind of experiment is possible, but has three main drawbacks. First, an APT specimen represents a TEM sample of inhomogeneous sample thickness and very limited volume. The latter issue is problematic if the interface structure needs to be analyzed at a more global scale, i.e., extending over a wider field of view, which is often required for arriving at statistically relevant structure-property relationships. Second, performing HRSTEM imaging on an APT specimen always carries the risk of beam damage and C contamination, especially when working with high acceleration voltages. Third, there is a higher risk that APT samples, that have undergone prior analysis by HRSTEM, break during the field evaporation process in the APT.
Despite these drawbacks, it is highly desirable to analyze one interface portion jointly with highest possible chemical and structural resolution. Therefore, we present here as proof of principle the possibility of imaging an interface inside an APT specimen in HRSTEM with atomic resolution. Several HAADF STEM micrographs of an APT specimen were taken at different magnifications in Figure 7. The needle-shaped volume contains a $\Sigma 3$ GB at a distance of $70 \mathrm{~nm}$ from the apex. Despite the inhomogeneous sample thickness and the comparatively thick sample with respect to the previous discussed TEM foils, the STEM images are of good quality and single atomic columns can be identified. As can be seen in Figures $7 b-7 d$ the main part of the observed GB consists of a (112) interface, and a smaller faceted section of alternating (112) and (111) facets. Unfortunately, the subsequently performed APT measurement failed and no chemical information of this specific GB exists.

\section{CONCLUSIONS}

In conclusion, we demonstrated the advantages of a combined sample preparation for joint APT and HRSTEM analysis. We were able to successfully apply the correlative 
approach at two different GB arrangements in mc-Si. In case of a $\Sigma 3-\Sigma 9-\Sigma 27$ TJ, the HAADF STEM images could resolve the complex atomic structure of the $\Sigma 9$ and $\Sigma 27$ interface. The $\mathrm{C}$ segregation in the correlated APT measurement suggests that the observed dissociation of the $\Sigma 27$ interface facilitates impurity segregation and thus leads to an increased recombination activity in the related EBIC signal.

In case of an incoherent $\Sigma 9$ interface, the APT data could only be interpreted together with the additional HRSTEM information. Here, two different GB segments with a fundamental different atomic structure were present, explaining the differences in the solubility of $\mathrm{C}, \mathrm{Cu},(\mathrm{Fe}+\mathrm{N})$, and $\mathrm{O}$ at these two interfaces. As a proof of principle, the faceted atomic structure of a $\Sigma 3$ GB inside an APT specimen was resolved in HRSTEM.

\section{ACKNOWLEDGMENTS}

The authors would like to thank Winfried Seifert for providing the mc-Si samples and for performing the quantitative EBIC analysis. The authors would also like to thank Uwe Tezins and Andreas Sturm for technical support running the APT and FIB systems at the MPIE. Part of this work was financed by the Federal Ministry of Education and Research (BMBF 03 X 5522A).

\section{REFERENCES}

Babinsky, K., De Kloe, R., Clemens, H. \& Primig, S. (2014). A novel approach for site-specific atom probe specimen preparation by focused ion beam and transmission electron backscatter diffraction. Ultramicroscopy 144, 9-18.

Batson, P.E., Dellby, N. \& Krivanek, O.L. (2002). Sub-angstrom resolution using aberration corrected electron optics. Nature 418(6898), 617-620.

Blavette, D., Wang, H., Bonvalet, M., Hüe, F. \& Duguay, S. (2014). Atom-probe tomography study of boron precipitation in highly implanted silicon. Phys Status Solidi A 211(1), 126-130.

Brandon, D.G. (1966). Structure of high-angle grain boundaries. Acta Metall 14(11), 1479-1484.

Cojocaru-Mirédin, O., Mangelinck, D. \& Blavette, D. (2009). Nucleation of boron clusters in implanted silicon. J Appl Phys 106(11), 113525.

Coullard, M., Radtke, G. \& Botton, G.A. (2013). Strain fields around dislocation arrays in a $\Sigma 9$ silicon bicrystal measured by scanning transmission electron microscopy. Philos Mag 93(10-12), 1250-1267.

Dabrowski, J. \& Müssig, H.-J. (2000). Silicon Surfaces and Formation of Interfaces: Basic Science in the Industrial World. World Scientific, Singapore.

Di Sabatino, M. \& Stokkan, G. (2013). Defect generation, advanced crystallization, and characterization methods for high-quality solar-cell silicon. Phys Status Solidi A 210(4), 641-648.

Felfer, P.J., Alam, T., Ringer, S.P. \& Cairney, J.M. (2012). A reproducible method for damage-free site-specific preparation of atom probe tips from interfaces. Microsc Res Tech 75(4), 484-491.

Herbig, M., Choi, P. \& RaAbe, D. (2015). Combining structural and chemical information at the nanometer scale by correlative transmission electron microscopy and atom probe tomography. Ultramicroscopy 153(0), 32-39.
Herbig, M., Raabe, D., Li, Y.J., Choi, P., Zaefferer, S. \& Goto, S. (2014). Atomic-scale quantification of grain boundary segregation in nanocrystalline material. Phys Rev Lett 112(12), 126103.

Istratov, A.A., Buonassisi, T., McDonald, R.J., Smith, A.R., SchindleR, R., Rand, J.A., Kalejs, J.P. \& Weber, E.R. (2003). Metal content of multicrystalline silicon for solar cells and its impact on minority carrier diffusion length. J Appl Phys 94(10), 6552-6559.

KäshAMmer, P. \& SINNO, T. (2015). A mechanistic study of impurity segregation at silicon grain boundaries. J Appl Phys 118(9), 095301.

Krakauer, B.W. \& Seidman, D.N. (1993). Absolute atomic-scale measurements of the Gibbsian interfacial excess of solute at internal interfaces. Phys Rev B 48(9), 6724-6727.

Kuzmina, M., Herbig, M., Ponge, D., Sandlöbes, S. \& Raabe, D. (2015). Linear complexions: Confined chemical and structural states at dislocations. Science 349(6252), 1080-1083.

Kveder, V., Kittler, M. \& Schröter, W. (2001). Recombination activity of contaminated dislocations in silicon: A model describing electron-beam-induced current contrast behavior. Phys Rev B 63(11), 115208.

Langford, R., Huang, Y., Lozano-Perez, S., Titchmarsh, J. \& Petford-Long, A. (2001). Preparation of site specific transmission electron microscopy plan-view specimens using a focused ion beam system. J Vac Sci Technol B 19(3), 755-758.

Lefebvre, W., Hernandez-Maldonado, D., Moyon, F., Cuvilly, F., Vaudolon, C., Shinde, D. \& Vurpillot, F. (2015). HAADFSTEM atom counting in atom probe tomography specimens: Towards quantitative correlative microscopy. Ultramicroscopy 159((Pt 2), 403-412.

Ohno, Y., Inoue, K., Tokumoto, Y., Kutsukake, K., Yonenaga, I., Ebisawa, N., Takamizawa, H., Shimizu, Y., Inoue, K., Nagai, Y., Yoshida, H. \& TAKedA, S. (2013). Three-dimensional evaluation of gettering ability of Sigma $3\{111\}$ grain boundaries in silicon by atom probe tomography combined with transmission electron microscopy. Appl Phys Lett 103(10), 102102.

Philippe, T., De Geuser, F., Duguay, S., Lefebvre, W., CojocaruMirédin, O., Da Costa, G. \& Blavette, D. (2009). Clustering and nearest neighbour distances in atom-probe tomography. Ultramicroscopy 109(10), 1304-1309.

Pizzini, S., Acciarri, M. \& BinetTi, S. (2005). From electronic grade to solar grade silicon: Chances and challenges in photovoltaics. Phys Status Solidi A 202(15), 2928-2942.

Riepe, S., Reis, I.E., Kwapil, W., Falkenberg, M.A., Schön, J., Behnken, H., Bauer, J., Kreßner-Kiel, D., Seifert, W. \& Koch, W. (2011). Research on efficiency limiting defects and defect engineering in silicon solar cells-results of the German research cluster SolarFocus. Phys Status Solidi C 8(3), 733-738.

Rigutti, L., Blum, I., Shinde, D., Hernández-Maldonado, D., Lefebvre, W., Houard, J., Vurpillot, F., Vella, A., Tchernycheva, M., Durand, C., Eymery, J. \& Deconihout, B. (2014). Correlation of microphotoluminescence spectroscopy, scanning transmission electron microscopy, and atom probe tomography on a single nano-object containing an InGaN/GaN multiquantum well system. Nano Lett 14(1), 107-114.

Schaffer, M., Schaffer, B. \& Ramasse, Q. (2012). Sample preparation for atomic-resolution STEM at low voltages by FIB. Ultramicroscopy 114, 62-71.

Stoffers, A., Cojocaru-Mirédin, O., Seifert, W., Zaefferer, S., RiePE, S. \& RAABE, D. (2015a). Grain boundary segregation in multicrystalline silicon: correlative characterization by EBSD, EBIC, and atom probe tomography. Prog Photovolt Res Appl 23(12), 1742-1753. 
Stoffers, A., Ziebarth, B., Barthel, J., Cojocaru-Mirédin, O., ElsÄsser, C. \& RaABe, D. (2015b). Complex nanotwin substructure of an asymmetric $\Sigma 9$ tilt grain boundary in a silicon polycrystal. Phys Rev Lett 115(23), 235502.

Thompson, K., Booske, J.H., Larson, D.J. \& Kelly, T.F. (2005). Three-dimensional atom mapping of dopants in Si nanostructures. Appl Phys Lett 87(5), 052108.

Thompson, K., Flaitz, P.L., Ronsheim, P., Larson, D.J. \& Kelly, T.F. (2007a). Imaging of arsenic cottrell atmospheres around silicon defects by three-dimensional atom probe tomography. Science 317(5843), 1370-1374.

Thompson, K., Lawrence, D., Larson, D.J., Olson, J.D., Kelly, T.F. \& GoRman, B. (2007b). In situ site-specific specimen preparation for atom probe tomography. Ultramicroscopy 107(2-3), 131-139.
Thuvander, M., Stiller, K., Blavette, D. \& Menand, A. (1996). Grain boundary precipitation and segregation in $\mathrm{Ni} \cdot 16$ Cr.9Fe model materials. Appl Surf Sci 94, 343-350.

Weber, J., Barthel, J., Brandt, F., Klinkenberg, M., Breuer, U., Kruth, M. \& Bosbach, D. (2016). Nano-structural features of barite crystals observed by electron microscopy and atom probe tomography. Chem Geol 424, 51-59.

Ziebarth, B., Mrovec, M., Elsässer, C. \& Gumbsch, P. (2015). Interstitial iron impurities at grain boundaries in silicon: A firstprinciples study. Phys Rev B 91(3), 035309. 